\title{
éditorial
}

\section{Maladies de pléthore}

En un siècle, la principale problématique nutritionnelle en France est passée des maladies de carence aux maladies de pléthore. L'obésité et le syndrome métabolique, le diabète et l'hypercholestérolémie guettent nos contemporains. Ces préoccupations se reflètent dans ce premier numéro de Médecine et Nutrition de l'année 2005. Au coeur du sujet, au coeur de la cellule adipocytaire, Max LAFONTAN et Michel BERLAN nous présentent les dernières connaissances sur les récepteurs adrénergiques de l'adipocyte, tandis que Anne-Marie ROUSSEL et Richard ANDERSON évoquent le rôle d'un oligo-élément, le chrome, dans le métabolisme énergétique : l'avenir nous dira si ces données de recherche fondamentale conduisent, ou non, à des avances significatives dans le domaine de la prise en charge de surpoids, mais quelques pistes apparaissent déjà.

Pour appréhender la prévention des maladies de pléthore, nous disposons certes de la recherche fondamentale, mais également des approches épidémiologiques, comme l'évoquent les travaux de l'équipe de Mongia GHARBI en Tunisie : mieux comprendre les évolutions des modes alimentaires est indispensable lorsqu'on cherche à être efficace en pratique, que ce soit en consultation ou dans une démarche de santé publique.

L'hypertension artérielle est elle aussi une maladie de civilisation. La responsabilité du sel dans sa genèse a bien sûr était évoqué, entre autres dans ces colonnes - voir Médecine et Nutrition $2004 n^{\circ} 1$-; à la lueur des travaux récents, le rôle préventif des fruits et des légumes apparaît lui aussi manifeste, comme nous l'explicitent Pierre MENETON et Christian RÉMÉSY.

$\grave{A}$ nous ensuite, praticiens et industriels, de mettre en place les stratégies préventives et thérapeutiques efficaces.

Jacques FRICKER 